\title{
TRIAGEM VIRTUAL PARA A IDENTIFICAÇÃO DE POTENCIAIS INIBIDORES DA PROTEASE SUBTILISINA 1 (SUB1) DO Plasmodium falciparum
}

\author{
Altino Caetano da Silva Neto ${ }^{1}$; Franco Henrique Andrade Leite ${ }^{2}$ \\ 1. Bolsista PIBIC/CNPq, Graduando em Farmácia, Universidade Estadual de Feira de Santana, e-mail: \\ altinocaetanoneto@gmail.com \\ 2. Orientador, Departamento de Saúde, Universidade Estadual de Feira de Santana, e-mail: fhpharm@gmail.com
}

PALAVRAS-CHAVE: Plasmodium falciparum; SUB1; Triagem virtual.

\section{INTRODUÇÃO}

A malária é uma doença infecciosa caracterizada por episódios febris, causada por protozoários do gênero Plasmodium ssp, a qual a espécie $P$. falciparum é responsável pela forma mais grave da doença, a cerebral (Blackman et al., 2012). Em 2015, essa doença foi responsável por cerca de 212 milhões de casos e 429 mil mortes em todo o mundo (WHO, 2015), sendo $99 \%$ delas causadas pelo P. falciparum. Apesar da relevância em termos de saúde pública, os fármacos disponíveis para o tratamento de pacientes com malária são limitados (quinolíncos e derivados da artemisinina), com efeitos adversos graves e eficácia espécie dependente e portanto a busca por novos fármacos antimalários é emergencial (2013; Sudhinaraset et al., 2016; Leite et al., 2014). Uma das formas para alcançar esse objetivo é através da utilização de estratégias computacionais que auxiliam na identificação de moléculas candidatas a ensaios biológicos frente a alvos exclusivos e validados do agente etiológico, como a Subtilisina 1 que é responsável por pela invasão e liberação dos parasitas do interior dos eritrócitos e, portanto, vital para o parasito (Brogi, 2016). Dentre essas técnicas, a triagem virtual por modelo farmacofórico e acoplamento molecular tem possibilitado a priorização de moléculas presentes em bibliotecas de moléculas com taxas de sucesso superior aos ensaios randômicos (Rodrigues et al, 2012). Com base nessas informações, triagem virtual hierárquica por modelo farmacofórico e acoplamento molecular foi utilizada com o objetivo de identificar potenciais inibidores frente a protease subtilisina 1 de P. falciparum (PfSUB1) nos bancos OOCC-UFSJ e Sigma-ZINC.

\section{METODOLOGIA}

Um conjunto de 37 inibidores não peptídicos com atividade biológica descrita frente à PfSUB1 (IC50 $<50 \mu \mathrm{M}$ ) foram coletados da literatura (Giovani, 2014) e posteriormente divididos em treino $(n=8)$ e teste para a geração e avaliação dos modelos farmacofóricos, respectivamente. As estruturas 2D foram obtidas no programa Marvin ${ }^{\circledR}$ Sketch 15.4.20 (Chemaxon, 2015, https://www.chemaxon.com/) e convertidas para o formato 3D na plataforma SYBYL®-X 2.0 (Tripos, 2010). Em seguida, as cargas parciais foram calculadas pelo método Gaisteiger-Huckel e as estruturas foram minimizadas por gradiente conjugado com um critério de convergência de $0,001 \mathrm{Kcal} / \mathrm{mol}$, utilizando campo de força Tripos conforme disponível na plataforma SYBYL®-X 2.0. Os modelos farmacofóricos foram gerados em duas etapas: Inicialmente, as conformações dos inibidores foram obtidas através de Algoritmo Genético implementado no módulo GALAHAD, no qual o tamanho da população e o número máximo de gerações para os inibidores de PfSUB1 foram ajustados para 50 e 10, respectivamente. Os demais parâmetros (CRUZAMENTO = 1,0 e MUTAÇÃO $=1,0$ ) foram mantidos nos seus valores padrão. Posteriormente, os confôrmeros foram alinhados entre si para a construção dos modelos farmacofóricos.

Inicialemnte, os modelos farmacofóricos foram avaliados quanto aos parâmetros estatísticos internos do GALAHAD (ENERGY $<100 \mathrm{kcal} / \mathrm{mol}$ e PARETO $\neq 0$ ). Os modelos remanescentes foram avaliados quanto a capacidade de identificar as moléculas do conjunto treino (N_HITS). Modelos com N_HITS $>4$ (>50\% do conjunto treino) foram selecionados para a próxima avaliação. A seguir, o modelo com maior valor de MOL_QRY foi considerado como o mais adequado para a etapa de triagem virtual. 
O melhor modelo farmacofórico foi utilizado para filtrar as moléculas disponíveis no banco da Sigma-Aldrich (http://zinc15.docking.org/catalogs/sial/) implementado na plataforma ZINC ${ }^{15}$ e o banco OOCC-UFSJ. Essa etapa foi implementada por meio da opção "alinhamento flexível 3D”, disponível no módulo UNITY ${ }^{\circledR}$ 3D. A qualidade do alinhamento de cada molécula foi expressa pelo valor de QFIT que varia de 0 a 100, em que aquelas com valores maiores que média mais duas vezes o desvio padrão foram escolhidas para a filtragem por acoplamento molecular. A estrutura 3D de PfSUB1 foi obtida do banco de estruturas macromoleculares (http://www.rcsb.org/pdb/) (PDB: 4LVN). As moléculas de água foram removidas e átomos de hidrogênio foram adicionados às estruturas de forma a otimizar suas ligações de hidrogênio. A seguir, os resíduos ácidos e básicos tiveram seus $\mathrm{pKa}$ calculados, com o programa H++ (http://biophysics.cs.vt.edu/). Finalmente, as cargas atômicas parciais Gasteiger-Hückel (Gasteiger; Marsili, 1980) foram atribuídas aos resíduos da proteína com o auxilio do módulo Biopolymer disponível na plataforma SYBYL®-X 2.0 (Tripos, 2010).

As etapas de busca conformacional e avaliação das poses foram realizadas no


resíduos catalíticos (Asp372, His428 e Ser606/ cubo de arestas $20 \AA$ Å). A seguir, esses parâmetros foram testados quanto a habilidade de diferenciar ativos de falsos positivos (decoys) construídos com o auxlílio do servidor DUD-E (Mysinger, et al. 2012). O banco contendo todas as moléculas (ativos + falso positivo $=508$ ) foi avaliado no sítio ortosteríco e os dados de afinidade utilizados para a geração da curva ROC (do inglês, Receiver Operating Csharacteristic) e cálculo da AUC (do inglês, area under the curve). Por fim, as duas moléculas melhores ranqueadas foram avaliadas quanto ao perfil de interações intermoleculares com o auxílio do programa PLIP (https://projects.biotec.tu-dresden.de/plipweb/plip/).

\section{RESULTADO E DISCUSSÃO}

Como a quimioterapia é a única alternativa terapêutica para o tratamento de pacientes com malária, os esforços para a busca de novos fármacos é fundamental para o controle da doença e sobrevida dos pacientes. Uma forma de acelerar esse processo é a utilização de métodos computacionais que utilizem dados oriundos de inibidores conhecidos e estrutura 3D de alvos essenciais para a manutenção da vida do parasito. Por esse motivo, uma filtragem hierárquica por modelo farmacofórico e acoplamento foi executada para a seleção de potencias inibidores da PfSUB1. Inicialmente, 10 modelos farmacofóricos foram construídos e avaliados conforme os parâmetros estatísticos internos do GALAHAD (Tabela 1).

Tabela 1: Parâmetros internos dos modelos farmacofóricos não-peptídicos disponíveis no GALAHAD.

\begin{tabular}{|c|c|c|c|c|c|c|}
\hline Modelos & $\begin{array}{l}\text { ENERGY } \\
\text { (Kcal/mol })\end{array}$ & STERICS & HBOND & $\begin{array}{l}\text { MOL_QR } \\
\text { Y }\end{array}$ & PARETO & N_HITS \\
\hline 1 & 22,97 & 1291,40 & 253,40 & 54,15 & 0 & 2 \\
\hline 2 & 14,08 & 1215,70 & 251,00 & 55,26 & 0 & 5 \\
\hline 3 & 39,66 & 1289,60 & 254,00 & 38,45 & 0 & 0 \\
\hline 4 & 102,56 & 1356,80 & 258,20 & 22,92 & 0 & 3 \\
\hline 5 & 17,95 & 1248,00 & 245,60 & 62,44 & 0 & 4 \\
\hline 6 & 36,24 & 1238,00 & 249,00 & 70,38 & 0 & 5 \\
\hline 7 & 16,49 & 1140,20 & 256,50 & 40,77 & 0 & 0 \\
\hline 8 & 24,32 & 1308,70 & 243,60 & 42,11 & 0 & 5 \\
\hline 9 & 21,21 & 1154,20 & 266,70 & 23,69 & 0 & 4 \\
\hline 10 & 17,65 & 1235,80 & 246,10 & 39,20 & 0 & 2 \\
\hline
\end{tabular}

A análise dos valores de ENERGY (>100Kcal $/ \mathrm{mol})$ mostra que apenas o modelo 4 contém moléculas com ângulos torcionais distorcidos. A comparação estatística dos modelos (valor de PARETO) também não foi útil para reduzir o número de modelos. Por essa razão, a 
capacidade de recuperar as molécula que deram origem aos modelos foi avaliada através do valor de N_HITS. Apenas os modelos 2, 6 e 8 conseguem identificar mais de $50 \%$ das moléculas do conjunto treino. Contudo, esse parâmetro não foi suficiente para a seleção de um modelo para a etapa de triagem virtual. Assim, o grau de sobreposição das moléculas do conjunto treino aos modelos remanescentes foi avaliado através do valor de MOL_QRY, o qual possibilitou a seleção de um modelo farmacofórico adequado para a triagem virtual (Figura 1, modelo 6).



Figura 1: Melhor modelo farmacofórico para inibidores de PfSUB1. Azul: centros hidrofóbicos, verde: pontos aceitadores de ligação de $\mathrm{H}$, rosa: pontos doadores de ligação de $\mathrm{H}$, vermelho: centro positivos. O raio das esferas varia de acordo ao raio de tolerância calculado pelo GALAHAD. As distâncias e raios estão representadas em angstrons.

O modelo farmacofórico 6 possui quatro grupos aceptores de ligação de $\mathrm{H}$, três doadores de ligação de $\mathrm{H}$, seis centros hidrofóbicos e um centro positivo. Esses pontos farmacofóricos são encontrados em inibidores potentes, o que demostra a capacidade do modelo em identificar os prováveis requisitos para a inibição frente a PfSUB1. A filtragem pelo modelo, possibilitou a seleção de 35 moléculas presentes nos bancos com valores de QFIT > 47,61 e portanto foram destinados a triagem por acoplamento molecular. Como não existem inibidores co-cristalizados na estrutura 3D de PfSUB1, a eficiência do acoplamento molecular foi avaliado com base na curva ROC e cálculo da AUC (Fig. 2).

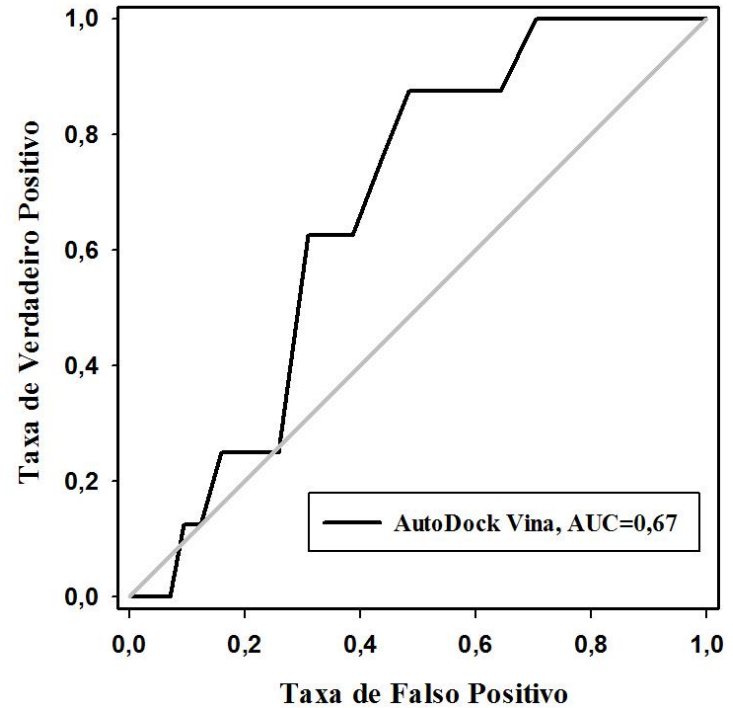

Figura 2: curva ROC da função de pontuação do acoplamento molecular

A literatura tem demostrado que programa que apresentam $\mathrm{AUC} \geq 0,65$ são considerados satisfatórios e, portanto podem ser uteis para a priorização de moléculas potenciais. Por esse motivo, 35 moléculas previamente filtradas foram acopladas no sítio ortosterico do alvo. A fim de avaliar o perfil de ligação, as duas moléculas com maior valor de afinidade (ZINC05940186; Energia $=-7 \mathrm{kcal} / \mathrm{mol}$ e ZINC00174021; Energia = -6,8 $\mathrm{kcal} / \mathrm{mol}$ ) (Fig. 3). 


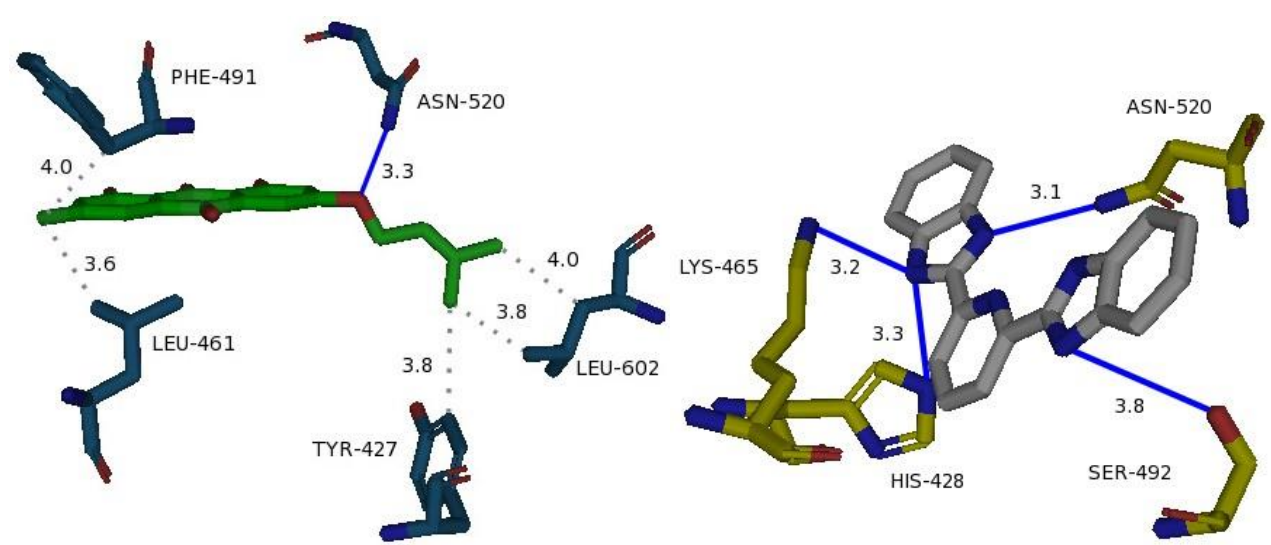

Figura 3: Mapa das interações intermoleculares das duas moléculas com maiores valores de afinidade. ZINC05940186 em verde e ZINC00174021 em branco. As ligações de hidrogênio estão representadas por linhas contínuas azuis, e as interações hidrofóbicas estão representadas pelas linhas tracejadas. As distâncias são expressas em angstrons.

As interações descritas por ZINC05940186 (Asp520, Leu461 e Leu602) e ZINC00174021 (His428, Lis465 e Asp520) são realizadas por resíduos catalíticos, o que pode demostrar potencial perfil de inibição e, portanto deve ser avaliadas por testes in vitro para confirmar a atividade biológica.

\section{CONCLUSÃO}

A escassez de inibidores não-peptídicos potentes (IC50 $<1 \mu \mathrm{M})$ e ausência de ligante cristalográfico dificultaram uma validação mais robusta paras técnicas empregas, contudo foi possível a construção de um modelo farmacofórico que pode conter características essenciais para inibição da PfSUB1 e filtragem de bancos moleculares, resultando em compostos que apresentaram interações com resíduos importantes do sítio ativo, que podem ser investigados em testes in vitro para avaliação de atividade biológica, corroborando também que as técnicas in silico são eficazes na priorização de moléculas que possivelmente possam conter atividade biológica.

\section{REFERÊNCIAS}

BLACKMAN, M. J. et al. Plasmodium subtilisinlike protease 1 (SUB1): Insights into the activesite structure, specificity and function of a panmalaria drug target. International Journal of Parasitology, [S.1], v. 42, p. 597-612, 2012.

BRASIL. Ministério da Saúde. Secretaria de Vigilância em Saúde. Boletim epidemiológico nacional, v. 46, n. 25, Brasília, DF, 2015.

BROGI, Simone et al. In silico study of subtilisin-like protease 1 (SUB1) from different Plasmodium species in complex with peptidyl-difluorostatones and characterization of potent pan-SUB1 inhibitors. Journal Of Molecular Graphics And Modelling, [s.1.], v. 64, p.121130, mar. 2016.

GIOVANI, Simone et al. Rational design of the first difluorostatone-based PfSUB1

inhibitors. Bioorganic \& Medicinal Chemistry Letters, [s.1.], v. 24, n. 15, 2014.

LEITE, F. H. A. et al. Malaria: from old drugs to new molecular targets. Biochemistry and

Biotechnology Reports. Londrina, v. 2, n.4, p. 1-7, 2014.

RODRIGUES, R.P. et al. Estratégias de Triagem Virtual no Planejamento de Fármacos.

Revista Virtual de Química, v. 4, n. 6, p. 739-776, 2012.

SONDERGAARD, C. R. et al. Improved Treatment of Ligands and Coupling Effects in Empirical Calculation and Rationalization of pKa Values. Journal of Chemical Theory and Computation, v. 7, n. 7, p. 2284-2295, 2011.

SUDHINARASET, M. et al. Who continues to stock oral artemisinin monotherapy? Results of a provider survey in Myanmar. Malarial Journal. v. 15, n. 1, 2016.

WORLD HEALTH ORGANIZATION. World Malaria Report 2015. Genebra, 2015. 